\section{Transmitter release statistics are meaningful}

BARTON and Cohen ${ }^{1}$ recently attempted to show that statistical analyses of neurotransmitter release, especially those based on a simple binomial model, are of dubious validity. Given their point of view, the authors might have asked: are any statistics meaningful? Every statistical analysis is based on certain assumptions, some purely formalistic, some containing empirical elements. If the statistician assumes an underlying binomial distribution then he tacitly assumes that there are a number of units, each of which may act independently in two ways with a certain probability for each way, the respective values for these probabilities being the same for all units. A binomial assumption was first satisfactorily applied to transmitter release at the crayfish neuromuscular junction ${ }^{2}$. In general terms such an analysis assumes units involving a certain class, $\mathrm{C}$, of probability distributions. The basic parameters of this class are (1) the form of dependence of the units; (2) the number of units, $n$; (3) the probabilities of release, $p_{1}, p_{2}$. . . p p $p_{n}$. The binomial is the simplest subclass of the whole with independence, $n$ constant and $p_{1}=p_{2} \ldots=p_{n}$. Each element of this subclass can be approximated in many ways by more complicated elements of $\mathrm{C}$, but is there any value in that when the simplest analysis suffices?

For example, in the case of falling bodies a random exponent, distributed about 2.00001 , would serve as well as the classic quadratic relation; orbits other than a simple ellipse could suffice to describe the path of the earth around the sun.

These examples illustrate the "principle of parsimony' which postulates that one's observations be described by as simple a model as possible. Experimental observations which cannot be described by the simplest model can then be used for gaining insight into the underlying processes in the real world and are described by, stepwise, more complex models (for example, the branch-block model proposed to explain experimental findings at the frog neuromuscular junction ${ }^{3}$ ).

The computed example of Barton and Cohen presents the result of a concatenation of binomial trials with a wide range of $p$ values. However, it is difficult to conceive that over 100 quanta with such low release probabilities $(p<0.01$ in over $70 \%$ of quanta) would result in so few failures of release (their Fig. $1 b$ shows only 2-3 failures). Nonetheless, in the result as presented, the simulated and predicted curves would seem to be different; indeed, the $\chi^{2}$ value quoted indicates that, had they simulated about 250 trials instead of 100 , a $5 \%$ level of significance would have been achieved with the same relative differences between the two histograms. This emphasises the importance of an adequate number of samples for satisfactory analysis of transmitter release.

We find the paper of Barton and Cohen a negative one, which makes no contribution to the real need, of which our own experimental results have made us particularly aware. This need is for a comprehensive theoretical treatment of particular generalisations of the binomial model, of which the branch-block model is simply one. Only then will interpretation of experimental results be soundly based; but without the experiments science can become merely a meaningless play with models.

\section{E. HANSERT \\ A. WERNIG \\ J. J. CARMODY \\ Max-Planck-Institut für Psychiatrie, D-8000 München 40, \\ Kraepelinstraße 2, FRG}

1. Barton, S. B. \& Cohen, I. S. Nature $268,267-268$ (1977).

2. Johnson. E. W. \& Wernig, A. J. Physiol., Lond. 218 , 757-767 (1971).

3. Carmody, J. J. \& Wernig, A. Pflügers Arch. ges. Physiol. R32 (1977).

Barton AND COHEN REPLY - We indicated $^{1}$ that many different $p$ distributions can lead to histograms of the quantal content which are indistinguishable from the binomial predictions. There are a number of reasons for rejecting the plea of Hansert, Wernig and Carmody that only the 'simplest analysis' should be considered. As we mentioned in our letter, the binomial assumption conflicts with at least two experimental observations. First, quantal contents greater than the binomial estimate of $n$ have been recorded at the crayfish neuromuscular junction; this is equivalent to a release of more than the total number of available quanta. Second, the timing of spontaneously released quanta is clustered, implying temporal or spatial non-uniformity of the release process. It is not obvious how the branch-block modification can explain this property of spontaneous release.

The implications of statistical analyses of quantal release are critically dependent

Table 1 The results of six simulations

\begin{tabular}{ccc}
\hline & $\begin{array}{c}\text { Degrees of } \\
\text { freedom }\end{array}$ & $P$ \\
5.421 & 7 & 0.61 \\
0.924 & 5 & 0.97 \\
6.921 & 6 & 0.34 \\
3.094 & 8 & 0.93 \\
8.172 & 6 & 0.23 \\
9.305 & 6 & 0.16 \\
\hline
\end{tabular}

The distribution of the release probability of the 100 quanta was generated in the same way as that in Fig. $1 a$ of our earlier letter ${ }^{1}$ $\left(\bar{z}=-5, \sigma_{z}{ }^{2}-2\right) .1,000$ trials were performed in each simulation. The $\chi^{2}$ value compares the simulated distribution of quantal contents with the best-fitting binomial distribution. The $P$ values give the probability that a value of $\chi^{2}$ greater than the observed value can arise by chance. on the assumed probability distribution. $n$ is generally assumed to represent an extensive property of the release process and $p$ an intensive one. However, if the binomial assumption is false then a change in the $p$ distribution of a fixed number of quanta would be interpreted as a change in both $n$ and $p$, or even as a change in $n$ alone.

The principle of parsimony is used to select the simplest of a set of equivalent hypotheses ${ }^{2}$. However, this principle is irrelevant because the predictions of the assumptions in question are different and experimental distinction of these hypotheses is possible.

We would like to clarify three other points raised by Hansert et al. First, there are only two failures of release when $70 \%$ of the quanta have $p<0.01$ because those quanta with high $p$ values effectively prevent large numbers of failures. If a single quantum had $p=1$ then no failures would ever occur. Second, we described a simulation of 100 trials-a sample size often used by some workers ${ }^{3}$ -but simulations of 1,000 trials produced similar results (see Table 1). It is incorrect to assume, as Hansert et al. did, that the relative shape of the quantal-content histogram remains the same when the sample size is increased. After publication of our letter it was brought to our attention that Brown, Perkel and Feldman ${ }^{4}$ had performed a similar analysis in 1976 and had reached the same conclusions that we did; they used 1,000 trials and studied the effects of temporal and spatial variations in $n$ or $p$. The distributions of quantal contents remained indistinguishable from the binomial predictions even at this high sample size. Third, we agree that a general theoretical treatment would be valuable, but the successful development of this seems unlikely without more data.

These arguments suggest to us that the assumption of binomial release is unlikely to be valid, despite its mathematical simplicity. We believe that the contribution of our letter can be determined only by its success or failure in stimulating new experiments designed to differentiate between the various hypotheses.

\section{Stuart B. Barton}

University Laboratory of Physiology, Oxford, UK

\section{IRA S. COHEN}

\section{Department of Physiology and Biophysics,}

Health Sciences Center,

SUNY, Stony Brook, USA

\footnotetext{
1. Barton, S. B. \& Cohen, I. S. Nature $268,267-268$ (1977).

Popper, K. R. The Logic of Scientific Discovery (Hutchinson, London, 1959).

Bennett, M. R. \& Fisher, C. J. Physiol., Lond. 271. 673-698 (1977)

4. Brown, T. H., Perkel, D. H. \& Feldman, M. W. Proc.natn. Acad. Sci. U.S.A. 73, 2913-2917 (1976).
} 